T. Zinno

Nagoya Math. J.

Vol. 33 (1968), 153-164

\title{
ON SOME PROPERTIES OF NORMAL MEROMORPHIC FUNCTIONS IN THE UNIT DISC
}

\section{TOSHIKO ZINNO}

1. We denote by $D$ the unit $\operatorname{disc}\{z ;|z|<1\}$ and by $\mathscr{S}$ the totality of one to one conformal mappings $z^{\prime}=s(z)$ of $D$ onto itself. A meromorphic function $f(z)$ in $D$ is normal if and only if the family $\{f(s(z))\}_{s(z) \in \mathscr{A}}$ is a normal family in $D$ in the sense of Montel. We denote by $\mathfrak{N}$ the totality of the normal meromorphic functions in $D$. Moreover, Noshiro introduced in [5] the notion of the normal meromorphic functions of the first category: $f(z)$ is a normal meromorphic function of the first category if and only if $f(z)$ belongs to $\mathfrak{R}$ and any sequence $\left\{f_{n}(z)\right\}$ obtained from the family $\{f(s(z))\}_{s(z) \in \mathscr{A}}$ can not admit a constant as a limiting function. We denote by $\mathfrak{R}_{1}$ the totality of the normal meromorphic functions of the first category. For instance, Schwarzian triangle functions belong to $\mathfrak{N}_{1}$. In $\S 1$, we shall give a necessary condition (Th. 1) and a sufficient condition (Th. 2) for a function to belong to $\mathfrak{N}_{1}$. Further we shall give some properties of a function of $\mathfrak{N}_{1}$. In these proofs the Hurwitz theorem will play an essential role.

In 1957, Lehto and Virtanen ([4]) showed that even if $f(z)$ and $g(z)$ belong to $\mathfrak{R}, f(z) \pm g(z)$ and $f(z) g(z)$ do not necessarily belong to $\mathfrak{R}$. Later Lappan ([2], [3]) gave sufficient conditions for $f(z) \pm g(z)$ and $f(z) g(z)$ to belong to $\Re$. In $\S 2$, we shall give a more general sufficient condition for $f(z) g(z)$ to belong to $\mathfrak{R}$ than that of Lappan.

\section{§1. Normal meromorphic functions of the first category}

2. We consider the hyperbolic distance

$$
d\left(z_{1}, z_{2}\right)=\frac{1}{2} \log \frac{\left|1-\bar{z}_{1} z_{2}\right|+\left|z_{1}-z_{2}\right|}{\left|1-\bar{z}_{1} z_{2}\right|-\left|z_{1}-z_{2}\right|}
$$

for $z_{1}$ and $z_{2}$ in $D$, and the chordal distance

$$
\chi(\alpha, \beta)=\frac{|\alpha-\beta|}{\sqrt{1+|\alpha|^{2}} \sqrt{1+|\beta|^{2}}}
$$

Received March 28, 1968. 
and

$$
\chi(\alpha, \infty)=\frac{1}{\sqrt{1+|\alpha|^{2}}}
$$

where $\alpha$ and $\beta$ are complex values. Put

$$
U(z, \delta)=\{\zeta ; d(z, \zeta)<\delta\} .
$$

We denote by $\bar{U}(\boldsymbol{z}, \boldsymbol{\delta})$ the closure of $U(\boldsymbol{z}, \boldsymbol{\delta})$.

Lemma 1. Let $f(z)$ be a function of $\mathfrak{R}_{1}$. Then so is

$$
\frac{a f(z)+b}{c f(z)+d} \quad(a d-b c \neq 0) .
$$

This follows immediately from the definition of $\mathfrak{R}_{1}$.

Lemma 2 (Noshiro [5]). Let $f(z)$ be a function of $\mathfrak{N}_{1}$. Then there exists a positive number $\rho_{0}$ such that for any point $z$ in $D, f(z)$ takes every value at least once in $U\left(z, \rho_{0}\right)$.

THEOREM 1. If $f(z)$ belongs to $\mathfrak{N}_{1}$, then $f(z)$ has the following three properties:

(i) There exist a positive number $\rho_{0}$ and a positive integer $q$ such that for any point $z$ in $D$ and every value $\alpha$,

$$
1 \leqq q(z, \alpha) \leqq q,
$$

where $q(z, \alpha)$ is the number of $\alpha$-points of $f(z)$ in $U\left(z, \rho_{0}\right)$.

(ii) For any two values $\alpha$ and $\beta(\alpha \neq \beta)$,

$$
\inf _{\substack{\nu=1,2,3, \ldots . \\ \mu=1,2,3, \ldots}} d\left(z_{\nu}(\alpha), z_{\mu}(\beta)\right)>0
$$

where $z_{\nu}(\alpha)$ and $z_{\mu}(\beta)$ denote $\alpha$-points and $\beta$-points of $f(z)$ respectively.

(iii) For any value $\alpha$ and any positive number $\rho$, there exists a positive number $m_{\rho}(<1)$ such that

$$
\chi(f(z), \alpha)>m_{\rho} \quad \text { in } \quad z \in D-\bigcup_{\nu=1}^{\infty} U\left(z_{\nu}(\alpha), \rho\right) .
$$

Proof of (i). Let $\rho_{0}$ be the same quantity in Lemma 2. Then $q(z, \alpha) \geqq 1$ for any point $z$ in $D$ and any value $\alpha$. Suppose that the set 
$\{q(z, \alpha) ; z \in D$ and $\alpha$ is an arbitrary value $\}$ is unbounded. There exist a sequence $\left\{z_{n}\right\}$ of points in $D$ and a sequence $\left\{\alpha_{n}\right\}$ of values such that

$$
\lim _{n \rightarrow \infty} q\left(z_{n}, \alpha_{n}\right)=\infty \text {. }
$$

Put

$$
f_{n}(z)=f\left(\frac{z+z_{n}}{1+\bar{z}_{n} z}\right)
$$

Since $f(z)$ belongs to $\mathfrak{R}_{1}$, there exist subsequences $\left\{f_{n_{k}}(z)\right\}$ of $\left\{f_{n}(z)\right\}$ and $\left\{\alpha_{n_{k}}\right\}$ of $\left\{\alpha_{n}\right\}$, a non-constant function $f_{0}(z)$ and a value $\alpha_{0}$ such that $\lim _{k \rightarrow \infty} \alpha_{n_{k}}=\alpha_{0}$ and $\left\{f_{n_{k}}(z)\right\}$ converges uniformly to $f_{0}(z)$ on each compact subset of $D$. Put

$$
g_{k}(z)=f_{n_{k}}(z)-\alpha_{n_{k}}, g_{0}(z)=f_{0}(z)-\alpha_{0} \text {, if } \alpha_{0} \neq \infty
$$

or

$$
g_{k}(z)=\frac{1}{f_{n_{k}}(z)}-\frac{1}{\alpha_{n_{k}}}, g_{0}(z)=\frac{1}{f_{0}(z)}, \text { if } \alpha_{0}=\infty .
$$

Then $\left\{g_{k}(z)\right\}$ converges uniformly to $g_{0}(z)$ on each compact subset of $D$. By the Hurwitz theorem, the number of zeros of $g_{k}(z)$ in $U\left(0, \rho_{0}\right)$ is not larger than that of $g_{0}(z)$ in $\bar{U}\left(0, \rho_{0}\right)$ for every sufficiently large $k$. On the other hand, since a transformation $s(z) \in \mathscr{S}$ preserves the hyperbolic distance, the former is equal to $q\left(z_{n_{k}}, \alpha_{n_{k}}\right)$. This contradicts (1. 1).

Proof of (ii). Suppose that there exist two values $\alpha$ and $\beta(\alpha \neq \beta)$ such that $\inf _{\nu=1,2,3, \ldots} d\left(z_{\nu}(\alpha), z_{\mu}(\beta)\right)=0$. Then there exist subsequences $\left\{z_{n}^{\prime}\right\}$ and $\left\{z_{n}^{\prime \prime}\right\}$ $\nu=1,2,3, \cdots$
$\mu=1,2,3, \cdots$

of $\left\{z_{\nu}(\alpha)\right\}$ and $\left\{z_{\mu}(\beta)\right\}$ such that

$$
\lim _{n \rightarrow \infty} d\left(z_{n}^{\prime}, z_{n}^{\prime \prime}\right)=0
$$

Put $f_{n}(z)=f\left(\frac{z+z_{n}^{\prime}}{1+\bar{z}_{3}^{\prime} z}\right), \zeta_{n}=\frac{z_{n}^{\prime \prime}-z_{n}^{\prime}}{1-\bar{z}_{n}^{\prime} z_{n}^{\prime \prime}}$ and $\zeta=s_{n}(z)=\frac{z+z_{n}^{\prime}}{1+\bar{z}_{n}^{\prime} z} \cdot$ By $\zeta=s_{n}(z), \quad 0$ and $\zeta_{n}$ correspond to $z_{n}^{\prime}$ and $z_{n}^{\prime \prime}$ respectively. Obviously $\lim _{n \rightarrow \infty} d\left(0, \zeta_{n}\right)=\lim _{n \rightarrow \infty} d\left(z_{n}^{\prime}, z_{n}^{\prime \prime}\right)=0$. Since $f(z)$ belongs to $\Re, \quad$ a subsequence $\left\{f_{n_{k}}(z)\right\}$ of $\left\{f_{n}(z)\right\}$ converges uniformly to a limiting function $f_{0}(z)$ on each compact subset of $D$. Therefore $\lim _{k \rightarrow \infty} f_{n_{k}}\left(\zeta_{n_{k}}\right)=\lim _{k \rightarrow \infty} f_{n_{k}}(0)=f_{0}(0)$. On the other hand, $f_{n_{k}}\left(\zeta_{n_{k}}\right)=f\left(z_{n_{k}}^{\prime \prime}\right)=\beta$ and $f_{n_{k}}(0)=f\left(z_{n_{k}}^{\prime}\right)=\alpha$. Hence $\alpha=\beta$; this 
is a contradiction.

Remark. As we see above, we can derive (ii) under the weaker condition $f(z) \in \mathfrak{R}$ than the condition $f(z) \in \mathfrak{R}_{1}$.

Proof of (iii). By Lemma 1, we may assume without loss of generality that $\alpha=0$. Let $\left\{a_{\nu}\right\}_{\nu=1}^{\infty}$ be all the zeros of $f(z)$ in $D$. Suppose that there exists a positive number $\rho$ such that

$$
\inf _{z \in D-\bigcup_{\nu=1}^{\infty} U\left(a_{\nu}, \rho\right)} \chi(f(z), 0)=0 .
$$

Then there exists a sequence $\left\{z_{n}\right\}$ of points in $D-\bigcup_{\nu=1}^{\infty} U\left(a_{\nu}, \rho\right)$ such that $\lim _{n \rightarrow \infty} f\left(z_{n}\right)=0$. Put $f_{n}(z)=f\left(\frac{z+z_{n}}{1+\bar{z}_{n} z}\right)$. Since $f(z)$ belongs to $\mathfrak{A}_{1}$, there exists a subsequence $\left\{f_{n_{k}}(z)\right\}$ of $\left\{f_{n}(z)\right\}$ converging uniformly to a nonconstant limiting function $f_{0}(z)$ on each compact subset of $D$. It holds

$$
f_{0}(0)=\lim _{k \rightarrow \infty} f_{n_{k}}(0)=\lim _{k \rightarrow \infty} f\left(z_{n_{k}}\right)=0 .
$$

Taking $\delta, 0<\delta<\frac{\rho}{2}$, sufficiently small, $f_{0}(z)$ has only one zero in $U(0, \delta)$. Let $m$ be its multiplicity. By the Hurwitz theorem, the number of zeros of $f_{n_{k}}(z)$ in $U(0, \delta)$ is equal to $m$ for every sufficiently large $k$. Namely, that of $f(z)$ in $U\left(z_{n_{k}}, \delta\right)$ must be equal to $m$ for every sufficiently large $k$. On the other hand, we took $\left\{z_{n_{k}}\right\}$ and $\delta$ such that $a_{\nu} \notin U\left(z_{n_{k}}, \delta\right)$ for all $\nu$ and all $k$. This is a contradiction. Thus the proof of Theorem 1 is complete.

3. The inverse of Theorem 1 also holds. In fact, we can give its proof assuming (i), (ii) and (iii) only for zeros and poles.

THEOREM 2. Let $f(z)$ be meromorphic in $D$. Suppose that $f(z)$ satisfies the following three conditions:

$(i)^{\prime}$ There exists a positive number $\rho_{0}$ such that $f(z)$ takes zero and $\infty$ at least once in $U\left(z, \rho_{0}\right)$ for any point $z$ in $D$.

(ii)' Let $a_{\nu}$ and $b_{\mu}$ be zeros and poles of $f(z)$ in $D$ respectively, then $\inf _{\substack{\nu=1,2,3, \ldots \\ \mu=1,2,3, \ldots}} d\left(a_{\nu}, b_{\mu}\right)>0$.

(iii)' For any positive number $\rho$, there exists a positive number $m_{\rho}$ such that 


$$
|f(z)|<m_{\rho} \quad \text { in } \quad z \in D-\bigcup_{\nu=1}^{\infty} U\left(b_{\nu}, \rho\right)
$$

and

$$
|f(z)|>\frac{1}{m_{\rho}} \quad \text { in } \quad z \in D-\bigcup_{\nu=1}^{\infty} U\left(a_{\nu}, \rho\right) .
$$

Then $f(z)$ belongs to $\mathfrak{R}_{1}$.

Proof. Take any sequence $\left\{s_{n}(z)\right\}$ out of $\mathscr{S}$. Put $f_{n}(z)=f\left(s_{n}(z)\right)$. For any fixed point $z_{0}$ in $D$, put $\zeta_{n}=s_{n}\left(z_{0}\right)$.

(a) If $\inf _{\substack{n=1,2,3, \ldots \\ \nu=1,2,3, \ldots}} d\left(\zeta_{n}, b_{\nu}\right)>0$, then $U\left(\zeta_{n}, \delta_{1}\right) \subset D-\bigcup_{\nu=1}^{\infty} U\left(b_{\nu}, \delta_{1}\right)$, where $0<\delta_{1}<\frac{1}{2} \inf _{\substack{n=1,2,3, \ldots \\ \nu=1,2,3, \ldots}} d\left(\zeta_{n}, b_{\nu}\right)$. By the condition (iii)', $f(z)$ is bounded in $U\left(\zeta_{n}, \delta_{1}\right)$ for $n=1,2,3, \cdots$ Hence $f_{n}(z)$ is also bounded in $U\left(z_{0}, \delta_{1}\right)$ for $n=1,2,3, \cdots$. Thus $\left\{f_{n}(z)\right\}$ is a normal family in $U\left(z_{0}, \delta_{1}\right)$.

(b) If $\inf _{\substack{n=1,2,3, \ldots \\ \nu=1,2,3, \ldots}} d\left(\zeta_{n}, b_{\nu}\right)=0$, then there exist subsequences $\left\{\zeta_{n_{k}}\right\}$ and $\left\{b_{\nu_{k}}\right\}$ of $\left\{\zeta_{n}\right\}$ and $\left\{b_{\nu}\right\}$ such that $\lim _{k \rightarrow \infty} d\left(\zeta_{n_{k}}, b_{\nu_{k}}\right)=0$. By the condition (ii)',

$$
\inf _{\substack{k=1,2,3, \ldots \\ \nu=1,2,3, \ldots}} d\left(\zeta_{n_{k}}, a_{\nu}\right)>0
$$

It holds that $U\left(\zeta_{n_{k}}, \delta_{2}\right) \subset D-\bigcup_{\nu=1}^{\infty} U\left(a_{\nu}, \delta_{2}\right)$, where $0<\delta_{2}<\frac{1}{2} \inf _{\substack{k=1,2,3, \ldots \\ \nu=1,2,3, \ldots}} d\left(\zeta_{n_{k}}, a_{\nu}\right)$. By the condition (iii)' there exists a positive number $m$ such that

$$
|f(z)|>\frac{1}{m} \text { in } z \in U\left(z_{n_{k}}, \delta_{2}\right) \text { for } k=1,2,3, \cdots,
$$

so that

$$
\left|f_{n_{k}}(z)\right|>\frac{1}{m} \text { in } z \in U\left(z_{0}, \delta_{2}\right) \text { for } k=1,2,3, \cdots
$$

Thus, $\left\{f_{n_{k}}(z)\right\}$ is also a normal family in $U\left(z_{0}, \delta_{2}\right)$. Therefore, there exists a subsequence $\left\{f_{m_{k}}(z)\right\}$ of $\left\{f_{n}(z)\right\}$ such that $\left\{f_{m_{k}}(z)\right\}$ converges uniformly to a limiting function on each compact subset of $D$. Since a transformation $s(z) \in \mathscr{S}$ preserves the hyperbolic distance, it is easy to see by the condition (i)' that any limiting function of the above normal family is nonconstant. The proof is now complete. 
4. Let $f(z)$ be meromorphic in $D$ and let $n(r, \alpha)$ be the number of $\alpha$-points of $f(z)$ in the domain $\{z ;|z|<r\}$.

Theorem 3. If $f(z)$ belongs to $\mathfrak{R}_{1}$, then there exist two positive numbers $A$ and $B$ such that for every $r$, sufficiently near 1 ,

$$
\frac{B}{1-r}<n(r, \alpha)<\frac{A}{1-r},
$$

where $A$ and $B$ are independent of the value $\alpha$.

To get Theorem 3, we need the following

Lemma 3. For positive numbers $r$ and $\rho$, with $0<r<1$ and $0<\rho<d(0, r)$, let $\theta$ be the positive angle formed by the real axis and the line segment, starting from the origin, tangent to the circle $d(r, z)=\rho$.

Then

$$
\sin \theta=\frac{\left(e^{4 \rho}-1\right)\left(1-r^{2}\right)}{4 e^{2 \rho} r}
$$

This is obtained by an elementary calculation.

Proof of Theorem 3. We shall first prove the left inequality of (1.2). Put

$$
\zeta_{n}=\frac{e^{2 n \rho_{0}}-1}{e^{2 n \rho_{0}}+1}
$$

and $R_{n}=\left\{z ;(2 n-1) \rho_{0} \leqq d(0, z)<(2 n+1) \rho_{0}\right\}$ for $n=1,2,3, \cdots$, where $\rho_{0}$ is the same quantity in (i) of Theorem 1. Let $m_{n}(\alpha)$ be the number of $\alpha$-points of $f(z)$ in $R_{n}$ and let $\theta_{n}$ be the positive angle formed by the real axis and the line segment, starting from the origin, tangent to the circle $d\left(\zeta_{2 n}, z\right)=\rho_{0}$. For any $r, \zeta_{3} \leqq r<1$, there exists a positive integer $N$ such that

$$
\zeta_{2 N+1} \leqq r<\zeta_{2 N+3}
$$

Obviously

$$
n(r, \alpha)>m_{N}(\alpha)
$$

In the ring domain $R_{N}$, we can take at least $\left[\frac{\pi}{\theta_{N}}\right]$ mutually disjoint open discs with a hyperbolic radius $\rho_{0}$, where [] denotes the Gauss sign. Therefore by (i) of Theorem 1, we have 


$$
m_{N}(\alpha) \geqq\left[\frac{\pi}{\theta_{N}}\right]>\frac{\pi}{\theta_{N}}-1>\frac{2}{\sin \theta_{N}}-1,
$$

and moreover, by Lemma 3

$$
=C \frac{e^{8 N \rho_{0}}-1}{e^{4 N \rho_{0}}}-1, \text { where } C=\frac{2 e^{2 \rho_{0}}}{e^{4 \rho_{0}}-1}
$$

Thus by combining (1. 3), (1. 4) and (1. 5)

$$
\begin{aligned}
&(1-r) n(r, \alpha)>\left(1-\zeta_{2 N+3}\right) m_{N}(\alpha) \\
&>\frac{2}{e^{(4 N+6) \rho_{0}}}+1 \\
&\left(C \frac{e^{8 N \rho_{0}}-1}{e^{4 N \rho_{0}}}-1\right) .
\end{aligned}
$$

It follows immediately that there exists a positive number $B$ such that

$$
n(r, \alpha)>\frac{B}{1-r}
$$

for every $r$, sufficiently near 1 , and that $B$ is independent of $\alpha$.

We shall now prove the right inequality of (1.2). Put $D(\rho)=\{z$; $d(0, z)<\rho\}, A(r)=\iint_{|z|<r} d \sigma(z)=\frac{\pi r^{2}}{1-r^{2}}$ and $s_{0}=\iint_{U\left(z, \rho_{0}\right)} d \sigma(z)$, where $d \sigma(z)=$ $\frac{r d r d \theta}{\left(1-r^{2}\right)^{2}}$. Since $d \sigma(z)$ is invariant by $s(z) \in \mathscr{S}, s_{0}$ is independent of $z$. Obviously, for any fixed value $\alpha$,

$$
D\left(d(0, r)+\rho_{0}\right) \supset \underset{z_{\nu}(\alpha) \in D(d(0, r))}{\bigcup} U\left(z_{\nu}(\alpha), \rho_{0}\right) .
$$

By (i) of Theorem 1, each point in the domain $D\left(d(0, r)+\rho_{0}\right)$ belongs to at most $q$-pieces of the open discs in $\left\{U\left(z_{\nu}(\alpha), \rho_{0}\right) ; z_{\nu}(\alpha) \in D(d(0, r))\right\}$. Hence it holds

$$
\begin{gathered}
q \underset{z \in D\left(d(0, r)+\rho_{0}\right)}{\iint_{z_{\nu}(\alpha) \in D(d(0, r))}} d \sigma(z) \geqq \int_{z \in U\left(\boldsymbol{z}_{\nu}(\alpha), \rho_{0}\right)} d \sigma(z) \\
q A\left(r^{\prime}\right) \geqq n(r, \alpha) s_{0},
\end{gathered}
$$

where $\quad r^{\prime}=\frac{e^{2 \rho_{0}}-1+r\left(e^{2 \rho_{0}}+1\right)}{e^{2 \rho_{0}}+1+r\left(e^{2 \rho_{0}}-1\right)}$.

We get immediately that

$$
\frac{A}{1-r} \geqq n(r, \alpha)
$$

where $A$ is a constant which is independent of $\alpha$. The proof of Theorem 3 is complete. 
Let $T(r, f)$ be the characteristic function of $f(z)$ in the sense of Nevanlinna. By Theorem 3 and Lehto and Virtanen ([4], p. 58), we shall get the following

CoRollary 1. If $f(z)$ belongs to $\mathfrak{R}_{1}$, then there exist two positive numbers $A^{\prime}$ and $B^{\prime}$ such that

$$
B^{\prime} \log \frac{1}{1-r}+O(1)<T(r, f)<A^{\prime} \log \frac{1}{1-r}+O(1)
$$

Corollary 2. If $f(z)$ belongs to $\mathfrak{R}_{1}$, then for any value $\alpha$,

(1) $\quad \sum_{\nu=1}^{\infty}\left(1-\left|z_{\nu}(\alpha)\right|\right)=\infty$

and

(2) $\sum_{\nu=1}^{\infty}\left(1-\left|z_{\nu}(\alpha)\right|\right)^{1+\lambda}<\infty$ for any positive number $\lambda$.

Proof of (1). For any value $\alpha$,

$$
\sum_{\nu=1}^{\infty}\left(1-\left|z_{\nu}(\alpha)\right|\right) \geqq \sum_{n=1}^{\infty} \sum_{z_{\nu}(\alpha) \in R_{n}}\left(1-\left|z_{\nu}(\alpha)\right|\right) \geqq \sum_{n=1}^{\infty}\left(1-\zeta_{2 n+1}\right) m_{n}(\alpha)
$$

By (1. 5)

$$
>\sum_{n=1}^{\infty} \frac{2}{e^{(4 n+2) \rho_{0}}+1}\left\{C \frac{e^{8 n \rho_{0}}-1}{e^{4 n \rho_{0}}}-1\right\}=\infty .
$$

Proof of (2). For any positive number $\lambda$ and any value $\alpha$,

$$
\begin{aligned}
\sum_{\left|z_{\nu}(\alpha)\right|<r}\left(1-\left|z_{\nu}(\alpha)\right|\right)^{1+\lambda} & =\int_{0}^{r}(1-t)^{1+\lambda} d n(t, \alpha) \\
& =(1-r)^{1+\lambda} n(r, \alpha)+(1+\lambda) \int_{0}^{r}(1-t)^{\lambda} n(t, \alpha) d t
\end{aligned}
$$

By Theorem 3

$$
\leqq A(1-r)^{\lambda}+A(1+\lambda) \int_{0}^{r} \frac{1}{(1-t)^{1-\lambda}} d t=O(1) .
$$

Hence $\sum_{\nu=1}^{\infty}\left(1-\left|z_{\nu}(\alpha)\right|\right)^{1+\lambda}<\infty$.

§2. Products of normal meromorphic functions

5. Theorem 4. Let $f(z)$ and $g(z)$ be two functions of $\Re$. Let $a_{\nu}$ and 
$a_{\nu}^{\prime}$ be zeros of $f(z)$ and $g(z)$ respectively and let $b_{\nu}$ and $b_{\nu}^{\prime}$ be poles of $f(z)$ and $g(z)$ respectively. Suppose that

(1) $\inf _{\substack{\nu=1,2,3, \ldots \\ \mu=1,2,3, \ldots}} d\left(a_{\nu}, b_{\mu}^{\prime}\right)^{*}>0$ and $\inf _{\substack{\nu=1,2,3, \ldots \\ \mu=1,2,3, \ldots}} d\left(a_{\nu}^{\prime}, b_{\mu}\right)>0$

and

(2) for any positive number $\rho$ there exists a positive number $m_{\rho}$ such that

$$
\begin{aligned}
& |f(z)|<m_{\rho} \quad \text { in } \quad z \in D-\bigcup_{\nu=1}^{\infty} U\left(b_{\nu}, \rho\right), \\
& |g(z)|<m_{\rho} \quad \text { in } \quad z \in D-\bigcup_{\nu=1}^{\infty} U\left(b_{\nu}^{\prime}, \rho\right), \\
& |f(z)|>\frac{1}{m_{\rho}} \quad \text { in } \quad z \in D-\bigcup_{\nu=1}^{\infty} U\left(a_{\nu}, \rho\right)
\end{aligned}
$$

and

$$
|g(z)|>\frac{1}{m_{\rho}} \text { in } z \in D-\bigcup_{\nu=1}^{\infty} U\left(a_{\nu}^{\prime}, \rho\right) .
$$

Then the product $f(z) g(z)$ belongs to $\mathfrak{R}$.

Proof. Take any sequence $\left\{s_{n}(z)\right\}$ out of $\mathscr{S}$. Put $f_{n}(z)=f\left(s_{n}(z)\right)$ and $g_{n}(z)=g\left(s_{n}(z)\right)$ for $n=1,2,3, \cdots$. Since $f(z)$ and $g(z)$ belong to $\mathfrak{R}$, it may be assumed without loss of generality that two sequences $\left\{f_{n}(z)\right\}$ and $\left\{g_{n}(z)\right\}$ converge uniformly to limiting functions $f_{0}(z), g_{0}(z)$ on each compact subset of $D$ respectively. For any fixed point $z_{0}$ in $D$, put $\zeta_{n}=s_{n}\left(z_{0}\right)$. We denote by $\delta_{1}$ the least value of $\inf _{\substack{\nu=1,2,3, \ldots \\ n=1,2,3, \ldots}} d\left(a_{\nu}, \zeta_{n}\right), \inf _{\substack{\nu=1,2,3, \ldots \\ n=1,2,3, \ldots}} d\left(b_{\nu}, \zeta_{n}\right), \inf _{\substack{\nu=1,2,3, \ldots \\ n=1,2,3, \ldots}} d\left(a_{\nu}^{\prime}, \zeta_{n}\right)$ and $\inf _{\substack{\nu=1,2,3, \ldots \\ n=1,2,3, \ldots}} d\left(b_{\nu}^{\prime}, \zeta_{n}\right)$.

(a) If $\delta_{1}>0$, then by the condition (2) there exists a positive number $m$ such that

$$
\frac{1}{m}<|f(z)|<m \text { and } \frac{1}{m}<|g(z)|<m \quad \text { in } \quad z \in U\left(\zeta_{n}, \frac{\delta_{1}}{2}\right)
$$

*) For two sequences $\left\{z_{n}\right\}$ and $\left\{z_{m}^{\prime}\right\}$ of points in $D$, we shall define $\inf d\left(z_{n}, z_{m}^{\prime}\right)=\infty$, if $\left\{z_{n}\right\}$ or $\left\{z_{m}^{\prime}\right\}$ is empty. 
for $n=1,2,3, \cdots$ Since $U\left(z_{0}, \frac{\delta_{1}}{2}\right)$ is mapped one to one comformally onto $U\left(\zeta_{n}, \frac{\delta_{1}}{2}\right)$ by $z^{\prime}=s_{n}(z)$, it holds

$$
\frac{1}{m^{2}}<\left|f_{n}(z) g_{n}(z)\right|<m^{2} \quad \text { in } z \in U\left(\overline{z_{0},} \delta_{1}\right) .
$$

Thus $\left\{f_{n}(z) g_{n}(z)\right\}$ is a normal family in $U\left(z_{0}, \frac{\delta_{1}}{2}\right)$.

(b) Suppose that $\delta_{1}=0$, say, $\inf _{\substack{\nu=1,2,3, \ldots \\ n=1,2,3, \ldots}} d\left(a_{\nu}, \zeta_{n}\right)=0$. There exist subsequences $\left\{a_{\nu_{k}}\right\}$ and $\left\{\zeta_{n_{k}}\right\}$ of $\left\{a_{\nu}\right\}$ and $\left\{\zeta_{n}\right\}$ such that

$$
\lim _{k \rightarrow \infty} d\left(\zeta_{n_{k}}, a_{\nu_{k}}\right)=0
$$

By Condition (1), $\delta_{2}=\inf _{\substack{k=1,2,3, \ldots . \\ \mu=1,2,3, \cdots}} d\left(a_{\nu_{k}}, b_{\mu}^{\prime}\right)>0 . \quad$ By Condition (2), $g(z)$ is bounded in $U\left(\zeta_{n_{k}}, \frac{\delta_{2}}{2}\right)$, so that $g_{n_{k}}(z)$ is bounded in $U\left(z_{0}, \frac{\delta_{2}}{2}\right)$ for every sufficiently large $k$. On the other hand, by (2.1)

$$
\lim _{k \rightarrow \infty} f\left(\zeta_{n_{k}}\right)=\lim _{k \rightarrow \infty} f\left(a_{\nu_{k}}\right)=0,
$$

so that $\lim _{k \rightarrow \infty} f_{n_{k}}\left(z_{0}\right)=\lim _{k \rightarrow \infty} f\left(\zeta_{n_{k}}\right)=0$. It follows that for every sufficiently large $k, f_{n_{k}}(z)$ is bounded in a neighborhood $U\left(z_{0}, \delta_{3}\right)$ of $z_{0}$. Put $\delta=\min$ $\left(\frac{\delta_{2}}{2}, \delta_{3}\right)$. The product $f_{n_{k}}(z) g_{n_{k}}(z)$ is bounded in $U\left(z_{0}, \delta\right)$ for every sufficiently large $k$. Thus $\left\{f_{n_{k}}(z) g_{n_{k}}(z)\right\}$ is a normal family in $U\left(z_{0}, \delta\right)$. Therefore, there exists a subsequence $\left\{f_{m_{k}}(z) g_{m_{k}}(z)\right\}$ of $\left\{f_{n}(z) g_{n}(z)\right\}$ such that $\left\{f_{m_{k}}(z) g_{m_{k}}(z)\right\}$ converges uniformly to a limiting function on each compact subset of $D$. The proof is complete.

6. The following Examples 1 and 2 show that Theorem 4 fails to hold without Condition (1) or Condition (2).

ExAmple 1. There exist two normal meromorphic functions $T_{1}(z)$ and $T_{2}(z)$ such that $T_{1}(z)$ and $T_{2}(z)$ satisfy Condition (2) but not Condition (1) and $T_{1}(z) T_{2}(z)$ does not belong to $\Re$.

To give this example, we need the following

Lemma 4. Let $d$ be an irrational number satisfying $0<d<1$. Then the set $\{n d-[n d]\}_{n=1}^{\infty}$ is dense on the closed interval $[0,1]$, where [] in \{\} denotes 


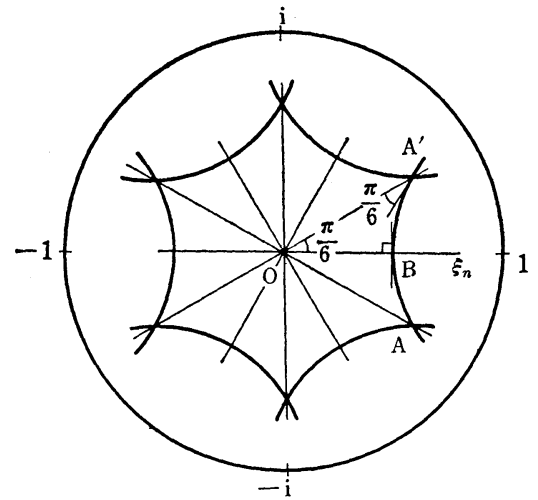

Fig. 1

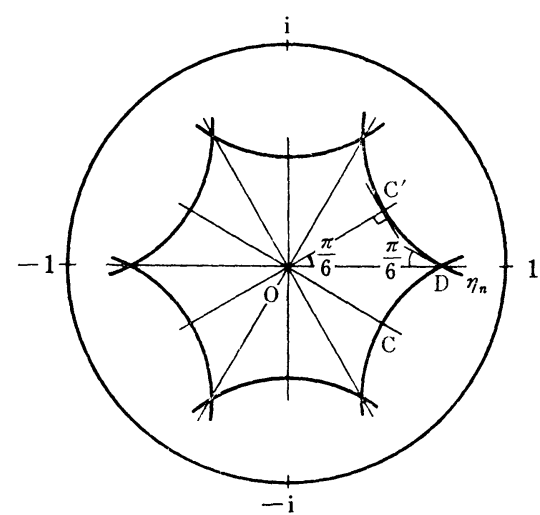

Fig. 2

the Gauss sign. (see G.H. Hardy and E.M. Wright [1], p. 155)

Let $T_{1}(z)$ and $T_{2}(z)$ be Schwarzian triangle functions whose fundamental triangles are shown in Figures 1 and 2 respectively. Let their system of triangles be those shown in [Fig. 1] for $T_{1}(z)$ and in [Fig. 2] for $T_{2}(z)$, where we assume $T_{1}(O)=0, T_{1}(A)=T_{1}\left(A^{\prime}\right)=\infty, T_{1}(B)=1, T_{2}(O)=\infty, T_{2}(C)=T_{2}\left(C^{\prime}\right)=0$ and $T_{2}(D)=1$. Then $T_{1}(z)$ and $T_{2}(z)$ belong to $\mathfrak{N}_{1}$, so that $T_{1}(z)$ and $T_{2}(z)$ satisfy Condition (2) by Theorem 1. Let $\xi_{n}$ and $\eta_{n}$ be zeros of $T_{1}(z)$ and poles of $T_{2}(z)$ on the segment $\{z=x+i y ; 0 \leqq x<1, y=0\}$ respectively. By an elementary calculation, we get $d\left(0, \xi_{n}\right)=n \log (\sqrt{2}+\sqrt{3})$ and $d\left(0, \eta_{n}\right)=$ $2 n \log (\sqrt{2}+1)$ for $n=1,2,3, \cdots$. Since $\frac{\log (\sqrt{2}+\sqrt{3})}{2 \log (\sqrt{2}+1)}$ is a positive irrational number less than 1 , it follows by Lemma 4 that the set

$$
\left\{n \log (\sqrt{2}+\sqrt{3})-2 \log (\sqrt{2}+1)\left[n \frac{\log (\sqrt{2}+\sqrt{3})}{2 \log (\sqrt{2}+1)}\right]\right\}_{n=1}^{\infty}
$$

is dense on the closed interval $[0,2 \log (\sqrt{2}+1)]$. Thus it is easy to see that there exist subsequences $\left\{\xi_{n_{k}}\right\}$ and $\left\{\eta_{n_{k}}\right\}$ of $\left\{\xi_{n}\right\}$ and $\left\{\eta_{n}\right\}$ such that

$$
\lim _{k \rightarrow \infty} d\left(\xi_{n_{k}}, \eta_{n_{k}}\right)=0
$$

Hence $T_{1}(z)$ and $T_{2}(z)$ do not satisfy Condition (1). The Product $\varphi(z)=T_{1}(z) T_{2}(z)$ does not belong to $\mathfrak{R}$. In fact, if $\varphi(z)$ belongs to $\mathfrak{R}$, then we must have by (2. 2)

$$
\lim _{k \rightarrow \infty} \varphi\left(\xi_{n_{k}}\right)=\lim _{k \rightarrow \infty} \varphi\left(\eta_{n_{k}}\right)
$$


On the other hand, $\varphi\left(\xi_{n_{k}}\right)=0$ and $\varphi\left(\eta_{n_{k}}\right)=\infty$. This is a contradiction.

Now we shall give our second example.

Lemma 5 (Lehto and Virtanen [4]). Let $f(z)$ be a function of $\mathfrak{R}$. If $f(z)$ has an asymptotic value $\alpha$, then the value $\alpha$ is an angular limit of $f(z)$.

EXAmple 2. Let $f(z)$ be an elliptic modular function and let $g(z)$ be a function of $\mathfrak{N}_{1}$. Then $f(z)$ and $g(z)$ satisfy Condition (1) because $f(z) \neq 0,1$ and $\infty$. But the product $f(z) g(z)$ does not belong to $\mathfrak{R}$.

In fact, let $e^{i \theta_{1}}$ be a point at which $f(z)$ has an angular limit $\infty$, let $a_{\nu}$ be zeros of $g(z)$, and let $\rho_{0}$ and $q$ the same quantities as those in Theorem 1. By Theorem 1, there exists a positive number $M$ such that

$$
|g(z)|>M \text { in } z \in D-\bigcup_{\nu=1}^{\infty} U\left(a_{\nu}, \frac{\rho_{0}}{3 q}\right) .
$$

Moreover, since the number of zeros of $g(z)$ in $U\left(z, \rho_{0}\right)$ is at most $q$ for every point $z$ in $D$, the point $e^{i \theta_{1}}$ is an accessible boundary point in the intersection $\tilde{\Delta}$ of the domain $D-\bigcup_{\nu=1}^{\infty} U\left(a_{\nu}, \frac{\rho_{0}}{3 q}\right)$ and a Stolz domain $\Delta$ at $e^{i \theta_{1}}$. Hence there exists a path $\Gamma$ ending at $e^{i \theta_{1}}$ in the domain $\tilde{\Delta}$, so that $\lim _{\substack{z \rightarrow e^{i \theta_{1}} \\ z \in \Gamma}} f(z)=\infty$. Therefore by (2.3) $\lim _{\substack{\boldsymbol{z} \rightarrow e^{i \theta_{1}} \\ z \in \Gamma}} f(z) g(z)=\infty$. If $f(z) g(z)$ belongs to $\Re$, then by Lemma $5 f(z) g(z)$ must have an angular limit $\infty$ at $e^{i \theta_{1}}$. On the other hand, since $g(z)$ has infinitely many zeros in the intersection of every neighborhood of $e^{i \theta_{1}}$ and the Stolz domain $4, f(z) g(z)$ can not possess an angular limit at $e^{i \theta_{1}}$.

\section{REFERENCES}

[1] G.H. Hardy and E.M. Wright, An introduction to the theory of numbers, Oxford 1938.

[2] P. Lappan, Non-normal sums and products of unbounded normal functions, Mich. Math. J., 8 (1961), 187-192.

[3] P. Lappan, Some sequential properties of normal and non-normal functions with applications to automorphic functions, Thesis. Univ. Notre Dame (1963), 41-57.

[4] O. Lehto and K.I. Virtanen, Boundary behavior and normal meromorphic functions, Acta Math., 97 (1957), 47-65.

[5] K. Noshiro, Contributions to the theory of meromorphic functions in the unit-circle, J. Fac. Sci. Hokkaido Univ., 7 (1938), 149-157.

Department of Mathematics, Mie University. 